\title{
Stratified layer in Martian mantle: Global thermochemical convection models
}

\author{
KAR WAI CHENG ${ }^{1}$, MAXIM D. BALLMER ${ }^{2}$, ANTOINE \\ ROZEL $^{3}$, GREGOR J. GOLABEK ${ }^{4}$ AND PAUL J. TACKLEY ${ }^{5}$ \\ ${ }^{1}$ ETH Zurich \\ ${ }^{2}$ University College London \\ ${ }^{3}$ Institute of Geophysics, Department of Earth Sciences, ETH \\ Zurich \\ ${ }^{4}$ University of Bayreuth \\ ${ }^{5}$ ETH Zürich \\ Presenting Author: karwai.cheng@erdw.ethz.ch
}

It has been suggested that the Martian mantle has gone through an early global magma ocean stage. The fractional crystallization of such a magma ocean and the overturn of the resulting $\mathrm{FeO}$ enriched top cumulate layer is thought to lead to stable mantle stratification, with a dense layer at the core-mantle boundary underlying the rest of the Martian mantle. This layer would be enriched in heat producing elements in addition to $\mathrm{FeO}$, thus having a significant impact on the thermal evolution of the planet.

We use the thermochemical convection code StagYY in a 2D spherical annulus geometry to model the long-term global geodynamical evolution of Mars following its magma ocean stage. We impose an initial dense primordial layer at the base of Mars' mantle to represent the final result of a convective overturn after magma-ocean crystallization [1]. By varying the primordial layer thickness as well as other global parameters, including reference viscosity, initial temperature and eruption efficiency, we systematically test the effect of different overturn scenarios. The modelled mantle temperature, lithosphere thickness and crust production are compared with observations, including petrological data, on Mars. It is shown that the primordial layer is likely molten, therefore seismic results may be sensitive to the presence of such a layer. In addition, the amount of crust produced by the Martian mantle with a primordial layer is significantly less than that without a layer in our models, implying the smaller amount of heat producing elements stored in the outer shell of the planet.

[1] Samuel, H., Ballmer, M., Padovan, S., Tosi, N., Rivoldini, A., \& Plesa, A. (2021) Journal Of Geophysical Research: Planets. e2020JE006613 